\title{
ABILITY OF MODAL ANALYSIS TO DETECT OSSEOINTEGRATION OF IMPLANTS IN TRANSFEMORAL AMPUTEES: A PHYSICAL MODEL STUDY
}

\author{
Nicola J. Cairns ${ }^{(1,2)}$, Mark J. Pearcy ${ }^{(1)}$ James Smeathers $^{(3)}$ Clayton J. Adam $^{(1)}$ \\ ${ }^{1}$ Institute of Health and Biomedical Innovation and School of Engineering Systems, Queensland University of \\ Technology, Brisbane, Australia \\ ${ }^{2}$ Department of Design, Manufacture and Engineering Management, University of Strathclyde, Glasgow, United \\ Kingdom. \\ ${ }^{3}$ Institute of Health and Biomedical Innovation and School of Exercise and Nutrition Sciences, Queensland University of \\ Technology, Brisbane, Australia.
}

\begin{abstract}
Owing to the successful use of non-invasive vibration analysis to monitor the progression of dental implant healing and stabilization, it is now being considered as a method to monitor femoral implants in transfemoral amputees. This study uses composite femur-implant physical models to investigate the ability of modal analysis to detect changes at the interface between the implant and bone simulating those that occur during osseointegration. Using electromagnetic shaker excitation, differences were detected in the resonant frequencies and mode shapes of the model when the implant fit in the bone was altered to simulate the two interface cases considered: firm and loose fixation. The study showed that it is beneficial to examine higher resonant frequencies and their mode shapes (rather than the fundamental frequency only) when assessing fixation. The influence of the model boundary conditions on the modal parameters was also demonstrated. Further work is required to more accurately model the mechanical changes occurring at the bone-implant interface in vivo, as well as further refinement of the model boundary conditions to appropriately represent the in vivo conditions. Nevertheless the ability to detect changes in the model dynamic properties demonstrates the potential of modal analysis in this application and warrants further investigation.
\end{abstract}

Keywords: Natural frequency, Resonant frequency, Vibration, Composite femur, Osseointegration, Transfemoral.

\section{INTRODUCTION}

Direct skeletal attachment of artificial limbs requiring osseointegration (OI) of the implant in the bone has been used in above knee (transfemoral) amputation for more than twenty years by the OPRA group in Sweden [15]. More recently alternative implant designs have been developed $[1,7,29]$ and implanted in amputees [1, 7]. Direct skeletal attachment, referred to as OI, offers an alternative to using a prosthetic socket, which is typically the interface between the amputee's residual limb and the prosthetic limb [27]. In transfemoral osseointegration (TFOI) developed by the OPRA group a threaded titanium implant is inserted into the medullary canal of the femur in the amputated limb. A second titanium component called an abutment connects to the implant and protrudes through the skin. When the implant is osseointegrated to the bone, prosthetic components can be attached to the distal end of the abutment without the need for a socket [31]. To date, over one hundred transfemoral amputees have been fitted with TFOI using the OPRA treatment in Europe, the U.K. and Australia [15].

TFOI has reported advantages over using a prosthetic socket. These include better control of the prosthetic limb and feedback through the limb, improvements in hip mobility and sitting comfort, fewer dermatological problems on the residual limb because of the elimination of the socket, and improved quality of life $[14,16]$. Consequently, TFOI can be a superior option for amputees who suffer from socket related problems, who have a residual limb that is too short for successful prosthetic socket use or who have an active lifestyle and require prosthetic limb function to match that lifestyle.

However, there are a number of disadvantages of TFOI. There is a risk of infection occurring at the skin protrusion site or implantation site and amputees can experience pain after surgery and during the rehabilitation exercises $[15,16]$. A major disadvantage of TFOI using the OPRA treatment is the lengthy rehabilitation. Due to the two stage surgical procedure and subsequent rehabilitation program, it takes between twelve and eighteen months from implant insertion for an amputee to be considered fully rehabilitated and able to load bear; a period of six months is allocated for the implant to integrate with the bone followed by six to twelve months of progressive rehabilitation [14]. TFOI amputees in the U.K. have commented that the rehabilitation program was longer than they originally expected and expressed frustration at the slow progress. They also included the high number of visits to the rehabilitation centre as a negative aspect of the program [30]. It is possible that the long rehabilitation time is the 
primary aspect of TFOI which potential candidates object to, and may be impeding the wider adoption of the technique as a realistic alternative to conventional socket prostheses. Therefore methods which aim to reduce the rehabilitation time require investigation.

If a non-invasive method was capable of assessing the development of OI between the bone and the implant and determining when the implant was able to withstand physiological load it could alter the overall rehabilitation time. Vibration analysis is a potential non-invasive method where changes detected in the dynamic properties of the bone-implant system could indicate changes in the physical properties at the interface between the bone and the implant throughout the progression of OI. Vibration studies of OI dental implants in vitro have demonstrated that changes in the implant interface condition (bone type, bone density, interface stiffness, exposed implant height) can be detected by measuring changes in the dynamic properties [13, 20, 21, 24]. Subsequent in vivo studies have verified the capability of vibration analysis to detect bone-implant interfacial changes during the progression of OI $[18,25]$. The success of the technique in dental applications has led to the development of two commercial devices (Osstell ISQ, Osstell AB, Gothenburg, Sweden; Periotest, Medizintechnik Gulden E.K, Modautal, Germany).

More recently vibration analysis has been extended to TFOI using physical models of the femur-implant system [28, 32]. Using an impact excitation technique, changes in the material stiffness of an interface region between a synthetic femur and implant and changes in the length of exposed implant were detected as shifts in the first natural frequency of the model. Using the same technique in a subsequent in vivo case study, changes in the first natural frequency were also reported as the rehabilitation exercises progressed. The authors concluded that the change in frequency in vivo indicated the improvement and stability of the implant boundary condition and that the technique had potential to be used as a reference during rehabilitation as well as for detecting OI failure [28].

While these studies provide a valuable initial assessment of the vibration technique for transfemoral osseointegration, they contain a number of methodological limitations which the current work attempts to address. Stainless steel implants were used in the in vitro laboratory tests, even though the clinical implants are titanium. Shao et al. acknowledged that the use of steel hinders the comparison of in vitro and in vivo measurements [28]. The method of constraining a model in laboratory tests is also important as the boundary conditions alter the natural frequencies obtained [6]; the previous studies held the model femur by clamping at the mid-span, which is arguably not analogous to the boundary condition of an amputated femur in vivo. Shao et al. chose to measure the system response and not the response and excitation. This methodology can only provide information about how the system behaves under the specific test conditions used [12]. Other dynamic properties inherent to the system which may be useful, for example the mode shape, are not obtained unless the excitation is measured. Furthermore, the frequency analysis was restricted to the first natural frequency. By contrast Delgado et al. measured four natural frequencies of a dental implant physical model and demonstrated that higher frequencies were more sensitive to certain interface parameters than the fundamental frequency [10]. Consequently, in the frequency analysis of the TFOI physical model, Shao et al. may have neglected higher frequencies that could be useful in indicating interface condition changes around the femoral implant.

Modal analysis using electromagnetic shaker excitation is considered by the authors to be a more appropriate vibration analysis methodology to investigate TFOI than impact because both excitation and response measurements can be performed over a large frequency range to provide multiple resonant frequencies and associated mode shapes of a structure [9]. Accordingly, this paper presents a modal analysis investigation using electromagnetic shaker excitation of physical models of the TFOI femur-implant system. The interface condition between the femur and the implant is altered and the models are tested using two different femur constraint arrangements to establish if the technique is capable of detecting interfacial changes for different femur boundary condition cases.

\section{METHODS}

\section{Physical Model Development}

Two physical models (Figure 1(a)) were developed using fourth generation large composite femurs (Sawbones model 3406, Pacific Research Laboratories Inc, WA, USA). Full details of the model development are provided in Cairns [8]. The composite femurs are made from an inner rigid polyurethane foam core (elastic modulus of $173 \mathrm{MPa}$, mass density of $270 \mathrm{~kg} / \mathrm{m}^{3}$ and Poisson's ratio of 0.26 ) and a short-fibre-filled epoxy outer shell (elastic modulus of $16 \mathrm{GPa}$, mass density of $1640 \mathrm{~kg} / \mathrm{m}^{3}$ and Poisson's ratio of 0.34 ) with a hollow canal through the polyurethane foam. Two composite femurs were cut to a length of $237 \mathrm{~mm}$ measured from the proximal end; the maximum amputation length recommended in TFOI surgery [31]. The canal of each femur was threaded using a Computer Numerical Control (CNC) machine to accommodate an implant. Fine adjustments were made iteratively to the female thread pitch of the canals so that one femur required an implant insertion torque of $4 \mathrm{Nm}$ and another of $0.5 \mathrm{Nm}$ representing a secure implant fit and a loose fit respectively. The $4 \mathrm{Nm}$ insertion torque and $0.5 \mathrm{Nm}$ insertion torque femur-implant systems were designed to represent extremes of the spectrum of implant integration with the bone in order to establish the ability of the modal analysis technique to detect gross changes in the interface between implant and femur. 
The implant and abutment become a rigid unit when assembled and tightened to the recommended torque value [31]. Therefore, the implant and abutment were modelled as a single component in this study (referred to hereafter as the implant) and this was not considered to alter the dynamic response of the system significantly. Two implants (Figure 1(c)) were machined from commercially pure titanium rod (elastic modulus of $115 \mathrm{GPa}$, mass density of $4511 \mathrm{~kg} / \mathrm{m}^{3}$ and Poisson's ratio of 0.3 ). The implants had a threaded section $80 \mathrm{~mm}$ long, $19 \mathrm{~mm}$ outer diameter and $1.75 \mathrm{~mm}$ male thread pitch. The profile of the implant then changed (matching the abutment profile) to a cylindrical section $60 \mathrm{~mm}$ long, $15 \mathrm{~mm}$ outer diameter. Flats were machined on the cylindrical section and $2.5 \mathrm{~mm}$ threaded holes were machined in the flats (Figure 1 (c)) to allow attachment of the excitation hardware. The implants were inserted into the sectioned composite femurs using a torque wrench so that the threaded section was embedded in the femur as in Figure 1(a)-(b) (sectioned femur mass, $0.3 \mathrm{~kg}$; implant mass, $0.1 \mathrm{~kg}$; total model mass of $0.4 \mathrm{~kg}$ ).

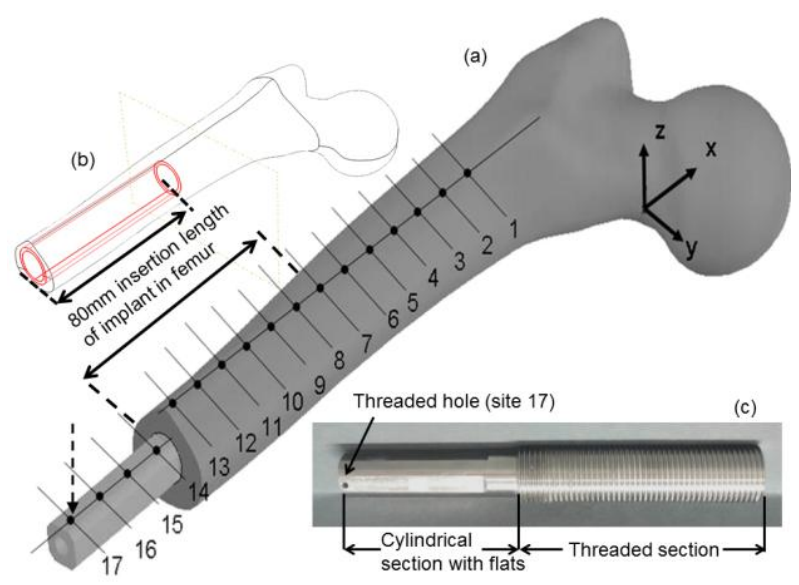

Fig.1: Diagram of (a) the femur-implant physical model, showing coordinate axes and numbered response sites for each model. Site 17 is the single excitation site used for each model, for example, the dashed arrow indicates z-direction excitation at the excitation site; (b) insertion length of the implant in the femur; (c) manufactured implant

\section{Boundary Conditions}

Two model boundary conditions were investigated; freely supported and cantilevered. In the freely supported case the physical models were supported on a soft foam bed as shown in Figure 2(a). The foam bed is commonly used experimentally to achieve freely supported boundary conditions [17, 22] and has been used successfully in the modal analysis testing of composite and cadaveric femurs [9, 11, 19]. After testing the models in the freely supported case they were constrained in a cantilever configuration. The femoral head was encapsulated in a block of Polymethylmethacrylate resin (Palapress, Heraeus Kulzer GmbH, Germany; elastic modulus of $2140 \mathrm{MPa}$ when cured) and then clamped. The cantilever boundary condition is discussed further in the discussion section. To create the resin block, the femur was fixed in a custom-made jig with a gap of $10 \mathrm{~mm}$ around the extremities of the femoral head. The two part cold-cure resin was mixed using the manufacturer recommended quantities and poured into the gap. After allowing the resin to cure for the manufacturer's stated time, the femur was removed from the jig. The resin block (dimensions $120 \times 75 \times 65 \mathrm{~mm}$ ) was clamped to a steel base (dimensions 500x510x25mm) fixed to the laboratory floor. Sections of $12 \mathrm{~mm}$ threaded rod were fitted through holes in the steel base and the resin block was fixed between the base and rectangular plates using nuts on the rods tightened to $16 \mathrm{Nm}$. The cantilever boundary condition is shown in Figure 2(b).

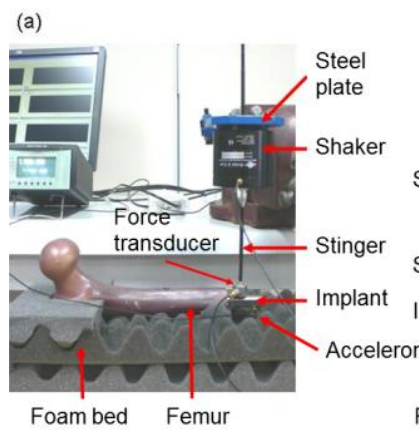

(b)

Fig.2: Model set up using (a) freely supported boundary conditions with shaker rigidly fixed to steel plate and (b) cantilevered boundary conditions with shaker suspended on spring

\section{Modal Analysis Methodology}

An electromagnetic shaker driven by a power amplifier (part numbers 4810 and 2706 Bruel\&Kjaer, Naerum, Denmark) was used to apply forced excitation to the models. The shaker methodology has been previously evaluated for this application using less complex models [9]. A signal generator (33120A, Agilent Technologies, CA, USA) input a sinusoidal sweep signal to the shaker. This excitation signal was measured using a dynamic force transducer $(0.028 \mathrm{~kg})$ powered by a signal conditioner (part numbers 2311500 and 4416B, Endevco, CA, USA). The sinusoidal sweep parameters $(100 \mathrm{~Hz}-10 \mathrm{kHz}$ frequency range, $500 \mathrm{mV}$ peak-to-peak amplitude and $5 \mathrm{kHz}$ per second sweep rate, 2 second duration) were optimised to obtain multiple resonant frequencies using a clinically acceptable low force level (maximum force measured using the force transducer was $4 \mathrm{~N}$; this is several orders of magnitude less than the force applied to the implant during typical TFOI rehabilitation exercises $[14,15])$ with adequate signal to noise ratio (defined as a coherence value of 0.8 or greater at resonance).

The shaker was connected to the force transducer via a Delrin stinger (Bruel\&Kjaer, Naerum, Denmark). The force transducer was connected to the model using a screw connection in the $2.5 \mathrm{~mm}$ threaded hole in the implant. The model response was measured using a 
single axis piezoelectric accelerometer $(0.002 \mathrm{~kg})$ connected to a charge conditioning amplifier (part numbers 4393 and 2692-A-0S2, Bruel\&Kjaer, Naerum, Denmark). The accelerometer was connected to the model using beeswax to allow its location to be easily changed. The excitation and response signals were recorded using a 16-bit resolution data logger (USB6259, National Instruments, NSW, Australia) connected to a personal computer (HP Intel ${ }^{\circledR}$ Core $^{\mathrm{TM}} 2 \mathrm{Duo} \mathrm{CPU}$ 3.5GB RAM) using data acquisition software (LabVIEW SignalExpress version 2.5, National Instruments) and a sampling rate of $50 \mathrm{kHz}$.

To maintain proper alignment of the shaker and the model, two shaker positions were used [23]. When testing the freely supported models the shaker was rigidly mounted to a steel plate (Figure 2(a)) and when testing the cantilevered models the shaker was suspended on a spring (Figure 2(b)). The steel plate had height and angle adjustment and, in both mounting arrangements, was used to align the shaker with the model.

A coordinate system and seventeen excitation/response measurement sites were identified along the length of the model femur as shown in Figure 1. Each model was excited ten times in the $\mathrm{z}$-axis direction by the sinusoidal signal at site 17 (dashed arrow in Figure 1(a)) with the accelerometer attached to response site 1 . The test was then repeated using the same excitation site but attaching the accelerometer to each response site (2-17) in turn (170 excitations in total). The shaker was then disconnected from the excitation site and the model rotated through 90 degrees to perform the test in the y-axis direction [9]. The shaker was reconnected to the excitation site in the yaxis direction and the seventeen tests were repeated. The $\mathrm{z}$-axis and $\mathrm{y}$-axis testing were conducted on the $4 \mathrm{Nm}$ and $0.5 \mathrm{Nm}$ femur-implant models first with the freely supported boundary condition and then repeated with the cantilever boundary condition.

\section{Data Analysis}

Customized analysis programs were written using MATLAB software (version 2007a, MathWorks Inc, Natick, MA, USA) to process the input and response signals. The MATLAB programs are detailed in Cairns [8]. Using a Fast Fourier Transform algorithm the time domain signals were converted to the frequency domain and the frequency response function (FRF), accelerance (defined as the ratio of acceleration response to excitation force) was computed. The accelerance function was calculated for the tests performed at each excitation/response site combination. These were used to compute a mean accelerance function for each physical model. Plots of the mean accelerance magnitude versus frequency were used to identify the resonant frequencies; resonant frequencies manifest as peaks on this graphical form of the FRF.

Another graphical form of the FRF was used to depict the mode shapes; a plot of the imaginary component of the accelerance versus frequency at each excitation/response site combination. A simplified description of obtaining mode shapes from this type of FRF plot can be found elsewhere in the literature [2, 3, 12]. In summary, the amplitude of the imaginary accelerance at a resonant frequency is comparative to the displacement magnitude occurring at that location (site) when vibrating in that mode, while the sign of the amplitude indicates the positive or negative direction of the displacement [3]. In addition, the value of imaginary accelerance away from resonances is close to zero. Therefore, by identifying the amplitude and the sign of the imaginary accelerance at each site (at a resonant frequency), the mode shape along the length of the femur-implant system can be determined. To start with the imaginary accelerance versus frequency plots calculated at each site were stacked together (forming a $3 \mathrm{D}$ plot) to visualise multiple mode shapes in one graph $[3,12]$. The plot was then zoomed to the first resonant frequency and viewed on the (2D) imaginary accelerance versus site plane to provide the mode shape associated with that resonant frequency. The mode shape depiction was simplified further by identifying the value and sign of the imaginary accelerance at each site and plotting on a line graph as in Figures 4 and 5. The process was repeated for each resonant frequency.

\section{RESULTS}

The z-axis and y-axis mean accelerance-frequency plots of the $4 \mathrm{Nm}$ and $0.5 \mathrm{Nm}$ femur-implant models are compared in Figure 3(a-d) for the freely supported and cantilevered boundary conditions. The upper limit of the frequency range plotted is $5 \mathrm{kHz}$. This limit was selected because a sufficient number of resonant frequencies were identified up to $5 \mathrm{kHz}$ to define differences in the two interface conditions and the experimental set-up used (in particular the use of beeswax to attach the response accelerometer to the SUT) produced superior signal to noise ratio up to $5 \mathrm{kHz}$.

The resonant frequencies (identified by the peaks in the accelerance-frequency plots) are indicated by the black, green and red arrows in Figure 3(a-d). A green arrow indicates a frequency identified for both the $4 \mathrm{Nm}$ and $0.5 \mathrm{Nm}$ models. These have been identified because a mode present at the same frequency in both interface models is unlikely to be suitable at identifying changes at the interface. The frequencies and mode shapes are also compared in Table 1, where possible the modes have been classified using the mode shape nomenclature of a uniform cross section beam with freely supported or cantilevered boundary conditions [6]. Reference to these classic mode shapes are in columns two and six of Table 1. The torsion mode in Table 1 refers to rotational motion of the femur-implant system about the long axis (x-axis in Figure 1(a)). Mode shapes that do not resemble a classic beam mode are identified as 'atypical'. 

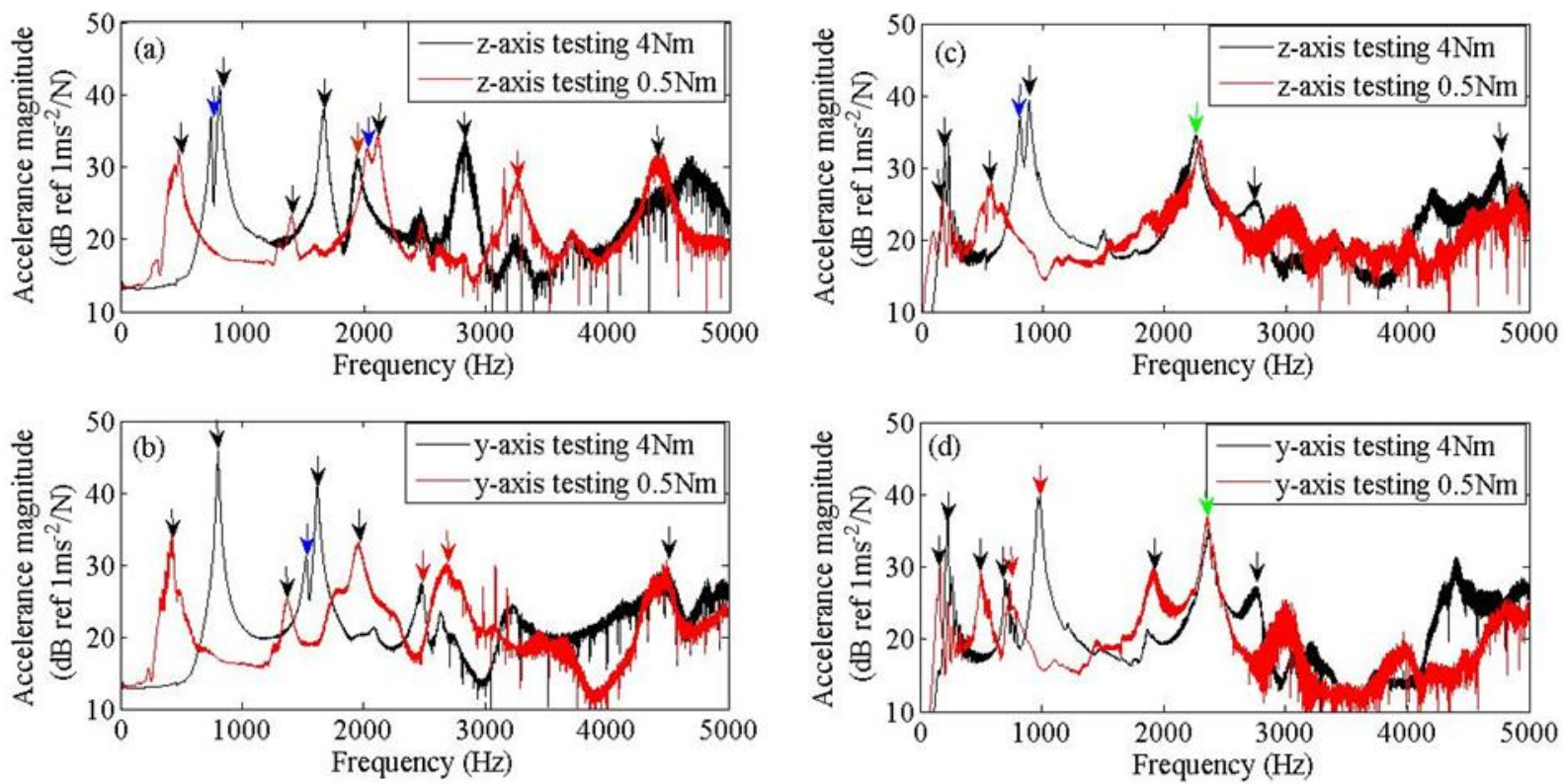

Fig.3: Mean accelerance functions for the freely supported models (a) and (b), and cantilevered models (c), (d). The green arrows indicate resonance seen in both $4 \mathrm{Nm}$ and $0.5 \mathrm{Nm}$ models; red arrows indicate the repeated second bending; black arrows indicate other resonant frequencies; blue arrows indicate a component of resonance from the orthogonal axis

Table 1: Comparison of resonant frequencies of $4 \mathrm{Nm}$ and $0.5 \mathrm{Nm}$ models for both boundary conditions and their approximate mode shapes. The percentage difference in frequency between the $4 \mathrm{Nm}$ and $0.5 \mathrm{Nm}$ model is defined as: $\%$ Diff $=((4 \mathrm{Nm}-0.5 \mathrm{Nm}) / 4 \mathrm{Nm}) * 100$

\begin{tabular}{|c|c|c|c|c|c|c|c|c|}
\hline & \multicolumn{4}{|c|}{ Freely supported } & \multicolumn{4}{|l|}{ Cantilevered } \\
\hline & \multicolumn{4}{|c|}{$\begin{array}{rr}4 \mathrm{Nm} & 0.5 \mathrm{Nm}\end{array}$} & \multicolumn{4}{|c|}{$4 \mathrm{Nm} \quad 0.5 \mathrm{Nm}$} \\
\hline & Mode shape & Frequ & $\mathrm{ccy}(\mathrm{Hz})$ & $\%$ Diff & Mode shape & Frequ & $\mathrm{icy}(\mathrm{Hz})$ & $\%$ Diff \\
\hline \multirow[t]{5}{*}{$\mathrm{z}$-axis } & $1^{\text {st }}$ bend & 814 & 476 & 42 & $1^{\text {st }}$ bend & 192 & 173 & 10 \\
\hline & $2^{\text {nd }}$ bend & 1669 & 1406 & 16 & $2^{\text {nd }}$ bend & 887 & 573 & 35 \\
\hline & atypical & - & 2111 & - & torsion & 2750 & - & - \\
\hline & $2^{\text {nd }}$ repeat & 1946 & 3255 & -67 & $4^{\text {th }}$ bend & 4768 & - & - \\
\hline & $3^{\text {rd }}$ bend & 2830 & 4419 & -56 & $3^{\text {rd }}$ bend & 2268 & 2299 & -1 \\
\hline \multirow[t]{6}{*}{$\mathrm{y}$-axis } & $1^{\text {st }}$ bend & 801 & 424 & 47 & $1^{\text {st }}$ bend & 230 & 165 & 28 \\
\hline & $2^{\text {nd }}$ bend & 1620 & 1375 & 15 & $2^{\text {nd }}$ bend & 700 & 505 & 28 \\
\hline & $2^{\text {nd }}$ repeat & 2480 & 2679 & -8 & $2^{\text {nd }}$ repeat & 990 & 739 & 25 \\
\hline & atypical & - & 1956 & - & atypical & - & 1924 & - \\
\hline & $3^{\text {rd }}$ bend & - & 4490 & - & $3^{\text {rd }}$ bend & 2373 & 2363 & 0.4 \\
\hline & & & & & torsion & 2750 & - & - \\
\hline
\end{tabular}

Owing to the complex geometry and non-uniform cross section of the femur-implant model, the modes are not purely directional [4] as in the case of a classical uniform cross section beam. Rather they often involve coupled displacements in both measurement axes (y and $\mathrm{z}$ axes defined in Figure 1). Some modes have motion principally in the y-axis with a smaller component of displacement occurring in the $\mathrm{z}$-axis and vice versa. Consequently the principally $y$-axis modes can be present in the accelerance function of the $\mathrm{z}$-axis testing (due to the $\mathrm{z}$-axis component of displacement) and vice versa [4]. This phenomenon can be seen in Figure 3(a)(c). Peaks with blue arrows in Figure 3(a) and (c) were considered to be the small z-axis component of principally y-axis modes (and vice versa for the blue arrow peak in Figure 3(b)). Therefore the peaks identified by blue arrows were not regarded as resonant frequencies in the specified testing axis. This conclusion was reached by comparing the mode shapes of each frequency peak. It was found that each blue arrow peak had the same mode shape as the larger magnitude peak adjacent to it. Therefore the larger magnitude peak was regarded as the principal mode in the direction of testing while the blue arrow peak was regarded as the small component of the same mode principally occurring in the orthogonal axis (therefore it is a resonant frequency in the orthogonal testing axis).

In the y-axis testing of all the models and the zaxis testing of the freely supported models, two frequencies were detected that both approximate the second bending mode of a classical beam $\left(2^{\text {nd }}\right.$ bend and $2^{\text {nd }}$ repeat in Table $1 ; 2^{\text {nd }}$ repeat indicated by red arrows 
in Figure 3(a), (b) and (d)) yet exhibit visible differences when compared to each other. The two such $\mathrm{z}$-axis mode shapes of the freely supported $4 \mathrm{Nm}$ model are illustrated in Figure 4. These were both considered resonant frequencies for two reasons. Firstly, they are both large magnitude peaks in the accelerance plot and secondly, they occur $277 \mathrm{~Hz}$ apart; an erroneous peak present in the FRF due to measurement errors or noise for example would be expected to have a lower magnitude and occur much closer to another resonant frequency [5]. The same reasoning was applied to the other models when evaluating similar modes indicated by the red arrows in Figure 3.

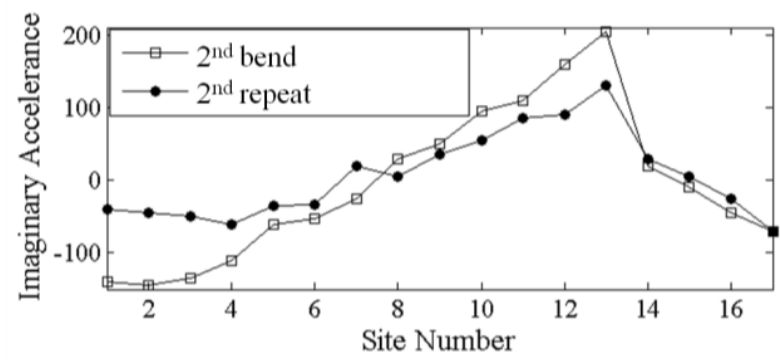

Fig.4: Comparison of two mode shapes of the $4 \mathrm{Nm}$ model with freely supported boundary conditions. Both resemble the classical beam second bending mode yet have different imaginary accelerance values at sites 113

It is evident from Figure 3 and Table 1 that for both freely supported and cantilever boundary conditions the resonant frequencies change when the implant fit is altered. The fundamental frequency was lower by $10-47 \%$ for the $0.5 \mathrm{Nm}$ insertion torque compared with the $4 \mathrm{Nm}$ insertion torque. Additionally, in the $\mathrm{z}$-axis testing a larger percentage change was found in the second, second repeat and/or third bending mode than in the fundamental frequency. Four modes (those not able to be related to classic beam modes and therefore labelled 'atypical' in Table 1) were only detected when the implant insertion torque was $0.5 \mathrm{Nm}$ (not at $4 \mathrm{Nm}$ ) and the torsional mode was only detected in the $4 \mathrm{Nm}$ cantilevered model (not at $0.5 \mathrm{Nm}$ ).

Implant insertion torque also caused changes in the bending modes. This is illustrated in Figure 5, by differences in the $y$-axis fundamental and second bending mode shapes for each model. The value of imaginary accelerance represents the relative magnitude and direction of displacement at each site. Thus, Figure 5 shows that the $4 \mathrm{Nm}$ models exhibit different deformation patterns to the $0.5 \mathrm{Nm}$ models. Also, there is an abrupt change in the magnitude of imaginary accelerance at implant sites 14-17.

The change in the frequencies and mode shapes due to the change from freely supported to cantilevered boundary conditions (compare columns 3 with 7 and 4 with 8 in Table 1; also compare Figure 3 (a) and (c)) is to be expected and is in accordance with beam vibration theory [10].

\section{DISCUSSION}

Modal analysis has been successfully used to assess implant osseointegration in dental applications, and the purpose of this study was to develop a physical model of the femur-implant system to assess the ability of the technique to detect changes in the system simulating stages of transfemoral osseointegration. The change in the resonant frequencies and mode shapes of the femurimplant model detected due to the change in implant fit within the femur demonstrate the potential of the modal analysis technique to assess progression of OI around the femoral implant. The positive results reported herein indicate the technique merits further development and evaluation.

The larger percentage change in frequency found in the second and/or third bending mode in the $\mathrm{z}$-axis testing compared to the fundamental frequency demonstrates the potential usefulness of the higher frequency modes in this application. It is possible that a higher frequency mode would prove to be the most sensitive mode in detecting interface condition changes occurring in vivo. Furthermore, over the specified frequency range some higher modes were only present in either the $4 \mathrm{Nm}$ or $0.5 \mathrm{Nm}$ model (in contrast to the fundamental frequency which of course is always detected). The identification of a particular mode other than the fundamental frequency within a specified frequency range and subsequently tracking the change in frequency of this mode may prove to be a successful method in assessing OI progression. The potential importance of higher resonant frequencies reported here is supported by the findings of Delgado et al. who discovered that the fourth frequency was the most sensitive mode to changes in the diameter of the hole around dental implants [10].

Differences in the deformation pattern of modes when comparing $4 \mathrm{Nm}$ and $0.5 \mathrm{Nm}$ models were found. In addition, two resonant frequencies with similar but not identical mode shapes (labeled $2^{\text {nd }}$ bend and $2^{\text {nd }}$ repeat in Table 1.) were detected for each model. It is possible that the mode shapes would continue to alter with different femur-implant interface conditions or that the presence and shape of an unusual mode would change with different interface conditions. Therefore, it is conceivable that the mode shapes themselves (rather than the frequency values) could be used to indicate the progression of OI.

In accordance with vibration theory the freely supported and cantilevered boundary conditions resulted in different resonant frequencies and mode shapes. This serves as a simple reminder that the boundary conditions applied to a physical model representing a musculoskeletal system should represent the in vivo constraints where possible. The freely supported boundary condition used in this study is regularly employed in modal analyses of structures [17, 22]. We note that the freely supported femur is not considered a realistic representation of the musculoskeletal boundary condition, but is used here in an iterative approach to evaluate the modal analysis set 


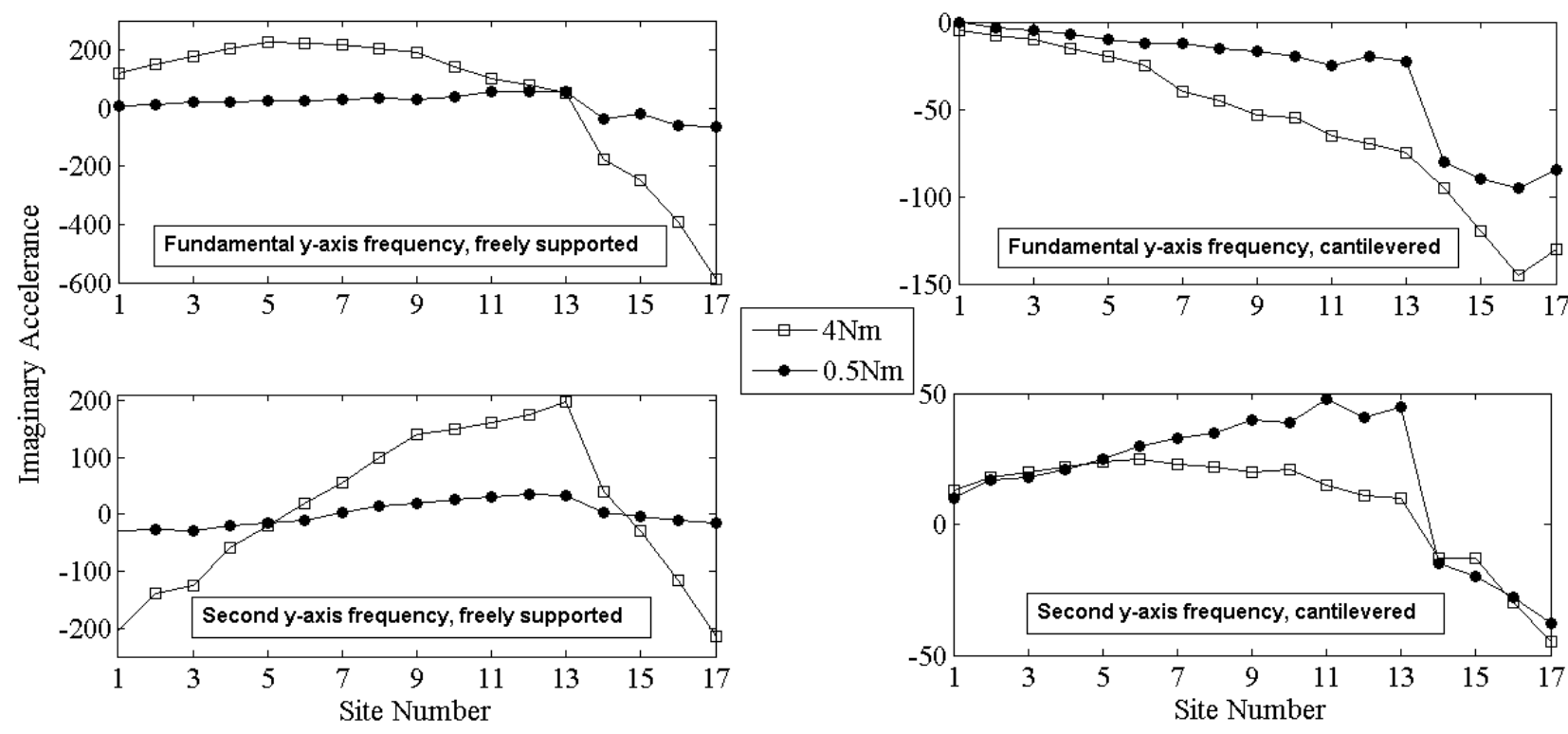

Fig.5: Comparison of y-axis fundamental and second frequency of the $4 \mathrm{Nm}$ and $0.5 \mathrm{Nm}$ models for both freely supported and cantilevered boundary conditions. Points where there is no motion at these frequencies (nodal points) are located where the mode shape crosses the zero imaginary axis

up, prior to progressing to more complex models, and also to compare the findings with earlier modal analyses of unmodified synthetic femurs that used the same boundary condition $[9,11]$.

The cantilevered boundary condition was developed as an approximate representation of the in vivo constraints on the femoral head of the amputated femur applied by the acetabulum and the muscle/soft tissue connections. A similar resin block boundary condition has been used in the modal analysis of the fractured tibia $[9,11,26]$ and therefore the cantilevered boundary condition was considered an acceptable first attempt at representing the in vivo conditions. However, considering the strong influence the boundary conditions have on the modal parameters obtained, further investigation is required to assess the impact of boundary condition changes on the measured differences in modal parameters due to interfacial changes representing the progression of TFOI.

It is primarily due to the different boundary conditions used between this study and Shao et al. that the results cannot be directly compared [28] . Nevertheless, a change in the interface condition was detected successfully using the modal analysis technique of the current study and this finding is in agreement with the results of Shao et al. Furthermore, the potential of using electromagnetic shaker excitation to detect higher frequency modes and/or mode shapes to indicate the progression of TFOI was demonstrated in the current study. This is not possible using the methodology of Shao et al. and identifies an advantage of using the current technique.

It is acknowledged that the representation of interfacial changes using different thread mating between the implant and the femur is an oversimplification of the likely interface changes occurring in vivo. In addition, the transition between $0.5 \mathrm{Nm}$ to
4Nm implant insertion torque provides a gross change in interface condition. Yet the two implant fits, intended to represent extremes of the spectrum of implant integration with the bone, were successfully used to establish the capability of the modal analysis technique in this application. Nevertheless, further investigation of alternative interface conditions which better attempt to mechanically represent the subtle changes occurring around the implant in vivo is required.

Using the current modal analysis set up in a clinical environment would be difficult. The length of time required to accurately attach the electromagnetic shaker and conduct the repeated tests make it impractical. Furthermore the shaft of the femur is not exposed in an amputated leg and therefore attaching the accelerometer directly to the bone would not be possible. Consequently due consideration needs to be given as to how to implement the technique clinically during its continued evaluation.

The capability of the modal analysis technique to detect changes in the interface condition of the femurimplant model for two boundary condition cases has been demonstrated. Not only were differences determined in the fundamental frequency of the model but also in the number of modes detected over a specified frequency range and in the mode shapes. In fact higher resonant frequencies were demonstrated to be more sensitive to implant fit than the fundamental frequency and therefore could prove useful in the detection of OI progression.

By repeating the modal analysis using two boundary condition cases, the impact of boundary conditions on the frequencies and mode shapes obtained was demonstrated. While the boundary conditions used here were considered appropriate in the early development stage of the modal analysis technique and as a first approximation of the in vivo boundary 
condition, further refinement and evaluation is necessary. Further refinement and evaluation of the interface condition is also required in order to better represent the musculoskeletal conditions in vivo. The positive findings reported here establish that the modal analysis technique warrants further investigation for the purpose of detecting TFOI progression.

\section{ACKNOWLEDGMENTS}

This work was funded by an Australian Government International Postgraduate Research Scholarship. The authors would like to thank the Built Environment and Engineering workshop staff at Queensland University of Technology for their technical assistance in the preparation of the physical models.

\section{REFERENCES}

1. Aschoff HH, Clausen A, Hoffmeister T (2009) The endo-exo femur prosthesis - a new concept of bone-guided, prosthetic rehabilitation following above-knee amputation. Z Orthop Unfall 147(5): 610-615

2. Avitabile P (1999) Modal space: back to basics. Exp Tech 23(3): 17-18

3. Avitabile P (1999) Modal space: back to basics. Exp Tech 23(5): 17-18

4. Avitabile P (2000) Modal space: back to basics. Exp Tech 24(3): 13-14

5. Avitabile P (2005) Modal space: back to basics. Exp Tech 29(6): 23-24

6. Blevins RD (1979) Straight beams. In: Yalof B (ed) Formulas for Natural Frequency and Mode Shape. Van Nostrand Reinhold Company, New York, pp 101-200

7. Blunn GW, Pendegrass CJ (2011) Development of an intraosseous transcutaneous amputation prostheses (Itap). In: 2011 MyoElectric Controls/Powered Prosthetics Symposium. , Fredericton, New Brunswick, Canada., pp

8. Cairns NJ (2010) The feasibility of vibration analysis as a technique to detect osseointegration of transfemoral implants. PhD Thesis, Queensland University of Technology, Brisbane, Australia

9. Cairns NJ, Adam CJ, Pearcy MJ, Smeathers J (2011) Evaluation of modal analysis techniques using physical models to detect osseointegration of implants in transfemoral amputees. In: Patton $\mathbf{J}$ (ed) Engineering in Medicine and Biology Society (EMBC), 2011 Annual International Conference of the IEEE, Boston, USA, pp 1600-1603

10. Delgado D, Jones R, Fuentes AA, Lumsdaine A (2002) Quantitative determination of the stability of implant-bone interface using resonance frequency analysis. In: ASME International Mechanical Engineering Congress and Exposition, New Orleans, pp 103-111

11. Dias Rodrigues JF, Lopes H, de Melo FQ, Simoes JA (2004) Experimental modal analysis of a synthetic composite femur. Exp Mech 44: 29-32
12. Dossing O (1988) Part 2 modal analysis and simulation. In: Bruel\&Kjaer (ed) Structural Testing. Bruel\&Kjaer, Naerum, pp 1-56

13. Elias JJ, Brunski JB, Scarton HA (1996) A dynamic modal testing technique for noninvasive assessment of bone-dental implant interfaces. J Oral Maxillofac Implants 11: 728-734

14. Hagberg K (2005) Physiotherapy for patients having a trans-femoral amputation. In: Chien S, Grondahl HG, Robinson K (eds) The Osseointegration Book From Calvarium to Calcaneus. Quintessenz Verlags-GmbH, Berlin, pp 477-487

15. Hagberg K, Brånemark R (2009) One hundred patients treated with osseointegrated transfemoral amputation prostheses - rehabilitation perspective. J Rehab Res Dev 46: 331-344

16. Hagberg K, Brånemark R, Gunterberg B, Rydevik B (2008) Osseointegrated trans-femoral amputation prostheses: Prospective results of general and condition-specific quality of life in 18 patients at 2-year follow up. Prosthet Orthot Int 32: $29-41$

17. He J, Fu ZF (2001) Frequency response function measurement. In: He J, Fu ZF (eds) Modal Analysis. Butterworth Heinemann, Oxford, pp 140-158

18. Huang HM, Cheng KY, Chen CF, Ou KL, Lin CT, Lee SY (2005) Design of a stability-detecting device for dental implants. Proc Inst Mech Eng $\mathrm{H}$ 219: 203-211

19. Khalil TB, Viano DC, Taber LA (1981) Vibrational characteristics of the embalmed human femur. J Sound Vib 75(3): 417-436

20. Lachmann S, Jäger B, Axmann D, Gomez-Roman G, Groten M, Weber H (2006) Resonance frequency analysis and damping capacity assessment. Part I: An in vitro study on measurement reliability and a method of comparison in the determination of primary dental implant stability. Clin Oral Implants Res 17: 75-79

21. Lachmann S, Laval JY, Jäger B, Axmann D, Gomez-Roman G, Groten M, Weber H (2006) Resonance frequency analysis and damping capacity assessment. Part 2: Peri-implant bone loss follow-up. An in vitro study with the periotest and osstell instruments. Clin Oral Implants Res 17: 8084

22. Maia NMM, Silva JMM, He J, Lieven NAJ, Lin RM, Skingle GW, To W-M, Urgueira APV (1997) Modal testing. In: Maia NMM, Silva JMM (eds) Theoretical and Experimental Modal Analysis. SRP Ltd, pp 123-184

23. Mayes RL, Gomez AJ (2006) Part 4: What's shakin', dude? Effective use of modal shakers. Exp Tech 30: 51-61

24. Meredith N, Alleyne D, Cawley P (1996) Quantitative determination of the stability of the implant-tissue interface using resonance frequency analysis. Clin Oral Implants Res 7: 261-267 
25. Meredith N, Boo K, Friberg B, Jemt T, Sennerby L (1997) Resonance frequency measurement of implant stability in vivo. Clin Oral Implants Res 8: 226-233

26. Nikiforidis G, Bezerianos A, Dimarogonas A, Sutherland C (1990) Monitoring of fracture healing by lateral and axial vibration analysis. $\mathrm{J}$ Biomech 23: 323-330

27. Schuch CM, Pritham CH (2004) Transfemoral amputation:Prosthetic management. In: Smith DG, Michael JW, Bowker JH (eds) Atlas of Amputations and Limb Deficiencies Surgical Prosthetic and Rehabilitation Principles. American Academy of Orthopaedic Surgeons, Rosemont, pp 541-555

28. Shao F, Xu W, Crocombe A, D E (2007) Natural frequency analysis of osseointegration for transfemoral implant. Ann Biomed Eng 35: 817-824

29. Shelton TJ, Beck JP, Bloebaum RD, Bachus KN (2011) Percutaneous osseointegrated prostheses for amputees: limb compensation in a 12-month ovine model. J Biomech 44: 2601-2606

30. Sullivan J, Uden M, Robinson KP, Sooriakumaran S (2003) Rehabilitation of the trans-femoral amputee with an osseointegrated prosthesis: The United Kingdom experience. Prosthet Orthot Int 27: 114-120

31. Ward DA, Robinson KP (2005) Osseointegration for the skeletal fixation of limb prostheses in amputations at the trans-femoral level. In: Chien S, Grondahl HG, Robinson K (eds) The Osseointegration Book From Calvarium to Calcaneus. Quintessenz Verlags-GmbH, Berlin, pp 463-476

32. $\mathrm{Xu} \mathrm{W}$, Shao F, Ewins D (2005) A resonant frequency measurement system for osseointegration trans-femoral implant. Key Eng Mater 295: 139-144 